\title{
Some Fundamental Limits on SAW RFID Tag Information Capacity and Collision Resolution
}

\author{
Richard J. Barton \\ Avionic Systems Division \\ NASA Johnson Space Center \\ Houston, TX USA \\ richard.j.barton@nasa.gov
}

\begin{abstract}
In this paper, we apply results from multi-user information theory to study the limits of information capacity and collision resolution for SAW RFID tags. In particular, we derive bounds on the achievable data rate per tag as a function of fundamental parameters such as tag time-bandwidth product, tag signal-to-noise ratio (SNR), and number of tags in the environment. We also discuss the implications of these bounds for tag waveform design and tag interrogation efficiency.
\end{abstract}

Keywords-SAW, RFID, Information Capacity, Collision Resolution

\section{INTRODUCTION}

For a wide range of application domains, remote sensing is a critical function that supports a diversity of activities such as environmental monitoring, planetary science, process control, structural shape and health monitoring, nondestructive evaluation, etc. The utility of the remote sensing devices themselves is greatly increased if they are "passive" - that is, they do not require any on-board power supply such as batteries - and if they can be identified uniquely during the sensor interrogation process. Additional passive sensor characteristics that enable greater utilization in specific applications such as space exploration are small size and weight, long read ranges with low interrogator power, ruggedness, and operability in extreme environments. In this paper, we consider one very promising passive sensor technology, called surface acoustic wave (SAW) radiofrequency identification (RFID), that satisfies all of these criteria.

In general, RFID is a method of identifying items using radio waves to interrogate "tags" encoded with a unique identifier that are affixed to the items of interest. In the case of passive tags, only the interrogator, which transmits power to the tags in the form of radio-frequency electromagnetic radiation, requires access to a power supply. Passive RFID technologies are used today in many applications, including asset tracking and management, security and access control, and remote sensing. To date, most of the development and application in RFID technology has focused on either asset/inventory tracking and control or security and access control because these are the largest commercial application areas. Recently, however, there has been growing interest in using passive RFID technology for remote sensing applications, and SAW devices are at the forefront of RFID sensing technology development [1-7].

Although SAW RFID tags have great potential for use in numerous remote sensing applications, the limited collision resolution capability of current generation tags limits the performance in a cluttered sensing environment. That is, as more SAW-based sensors are added to the environment, numerous tag responses are superimposed at the receiver and decoding all or even a subset of the telemetry becomes increasingly difficult. Background clutter generated by reflectors other than the sensors themselves is also a problem, as is multipath interference and signal distortion, but the limiting factor in many remote sensing applications can be expected to be tag mutual interference.

This problem can be greatly mitigated by proper design of the SAW tag waveform, even within the constraints imposed by the physical characteristics of SAW devices. In particular, results from coding theory and multi-user communication and information theory can be applied to develop tag encoding and decoding schemes that are superior to the more ad-hoc spread-spectrum techniques that are often utilized [8-10] and to derive performance bounds that can be utilized as benchmarks for design and performance evaluation. Such new, improved tag encoding and decoding techniques are currently under investigation at NASA Johnson Space Center (JSC), but are not discussed explicitly in this paper.

Instead, in this work, we explore the fundamental limits of tag data capacity and collision resolution. In particular, we apply results from multi-user information theory to derive bounds on the achievable data rate per tag as a function of parameters such as tag time-bandwidth product, tag signalto-noise ratio (SNR), and number of tags in the environment. We also discuss the implications of these bounds for tag waveform design, tag interrogation efficiency, and RFID sensor system performance evaluation. Finally, we include some simulation results with synthetic but realistic template waveforms which indicate that the theoretical limits on achievable data rates per tag lead to practical performance bounds that can be approached if tag waveforms and interrogator algorithms are properly designed. 


\section{BRIEF BACKGROUND ON SAW TAGS}

SAW RFID technology differs from more conventional integrated-circuit (IC) based RFID technology in many significant ways. For example, SAW RFID tags do not need to rectify incident electromagnetic power arriving at the tag. Instead, they modulate and re-radiate the interrogation signal directly using a series of reflectors printed on the surface of the device. The principle benefit of this approach is that SAW RFID tags are capable of operating at much lower interrogator transmit power, and/or are capable of much greater range for a given transmit power than IC-based tags [11]. In addition, SAW RFID tags have the capability to incorporate certain types of sensor telemetry along with the identification information. For example, the impulse response of a SAW RFID tag is sensitive to tag temperature and tag strain as well as more esoteric environmental parameters such as the presence of certain gasses. Hence, parameters such as temperature, acceleration, pressure, etc. can be estimated by comparing the tag impulse response to a reference waveform. Finally, SAW RFID tags are very robust and can tolerate extreme conditions that would render IC-based tags inoperable.

Consider the most common SAW sensing application, which is that of temperature measurement. As it turns out, temperature (as well as strain and other parameters) of a SAW RFID tag can be estimated by direct measurement of the time dilation (or contraction) of the tag impulse response. In particular, measurement of the time dilation of the impulse response at an arbitrary temperature relative to the response at a known reference temperature (e.g., $0^{\circ} \mathrm{C}$ ) constitutes an observation of the temperature coefficient of delay (TCD) for the tag at its current temperature. Here, the term TCD refers to the mathematical function of temperature that quantifies the relationship between the relative time dilation of the tag response and the temperature of the tag, with respect to a fixed reference temperature. Although the TCD can theoretically be determined from the piezo-electric properties of the crystalline material used to manufacture the tag, it is more common (and probably more accurate) to estimate it experimentally.

For further information on current SAW technology, an excellent review is given in [9].

\section{THEORETICAL RESULTS}

The results developed in this section are completely general and apply not only to SAW RFID tags but IC-based tags as well. However, since this paper is focused on SAW tags, the discussion has been tailored specifically to those tags.

The RFID interrogation problem is well modeled as a Gaussian multiple-access channel. That is, we assume that over some fixed time interval $t \in[0, T]$ following the transmission of an interrogator signal, all of the tags in the environment will respond by transmitting a unique ID signal within a fixed bandwidth $[-B / 2, B / 2]$ and that the signal received back at the interrogator during this time interval is the superposition of all tag responses plus complex-valued additive white Gaussian noise (AWGN) with power spectral density (PSD) of $N_{0}$ watts/Hz. Note that the complexvalued, baseband model adopted here is a reflection of the fact that real-valued signals can be modeled at baseband using complex-valued low-pass equivalent signals [12] with no loss of generality and with less mathematical complexity.

Within this very general context, we can determine fairly precisely the relationship between achievable bit-rate per tag (i.e., the number of bits of information reliably communicated by each tag), achievable collision resolution (i.e., how many tags can be simultaneously decoded reliably), time-bandwidth product ( $B T$ in this case), and signal-to-noise ratio ( $S N R=\mathcal{E}_{s} / N_{0}$, where $\mathcal{E}_{s}$ represents the energy received per tag at the reader). Note that the term "reliable" used above is taken from the underlying information-theoretic results used to derive the results presented here. In this context, the term reliable means that an arbitrarily low probability of tag ID decoding error can theoretically be achieved as long as the time-bandwidth product of the tags is allowed to go to infinity. For finite values of the time-bandwidth product, there will always be a non-zero probability of tag decoding error that is based on all of the parameters of the actual problem, but the fundamental relationships elucidated here between all of these parameters is extremely useful when attempting to determine what one can and cannot do with SAW RFID tags.

To derive the desired results without expressions or notation becoming cumbersome and somewhat of a distraction, we make the simplifying assumptions that the number $M$ of tags in the environment is known, that each tag response is received with identical energy $\mathcal{E}_{s}$ regardless of its range from the interrogator, that $\mathcal{E}_{\mathrm{s}}$ is also known, and that the bit-rate $R$ for all tags is identical. Under these assumptions, $R$ is an achievable bit-rate per tag if and only if it satisfies [13]

$$
R<\frac{B T}{M} \log _{2}\left(1+\frac{M \cdot S N R}{B T}\right)
$$

Equivalently, the maximum total number of tags with fixed bit-rate $R$ that can be reliabley decoded simultaneously is given by the largest integer satisfying

$$
M<\frac{B T}{R} \log _{2}\left(1+\frac{M \cdot S N R}{B T}\right) .
$$

Note that for the simple RFID problem, where the tag ID is the only quantity of interest and no telemetry data (such as temperature) are modulated on the tag response, the bit-rate per tag is just the logarithm, base 2, of the total number of tag IDs (i.e., the size of the tag constellation) for which the interrogator is scanning. Note also that modulation of the tag response due to environmental parameters such as temperature, strain, or range represents information encoded in the tag in addition to the tag ID, so the bit-rate per tag increases due to tag modulation just as if the length of the tag 
ID (equivalently, the size of the tag constellation) increased to represent measured data. This is essentially true whether the environmental modulation is of interest or not, so uncertainty regarding the exact structure of the received tag waveforms due to range, temperature, or other environmental parameters can severely degrade the achievable collision resolution for the tags by effectively increasing the bit-rate. Of course, the amount of information represented by waveform modulation parameters such as time delay or time dilation is limited by the precision with which such parameters can be estimated, which is in turn limited by SNR, so it is difficult to quantify precisely the effective bitrate per tag in the RFID sensing application. Finally, note that for practical purposes, the number $M$ of tags in the environment can be much smaller than the size of the tag constellation because interrogators can be programmed to scan for a relatively large number of possible tags even if it is known that the current environment contains only a subset of those tags.

There are several points of interest regarding Equations (1) and (2). First, the term " $R$ is an achievable bit-rate per tag" for a fixed time-bandwidth product and a fixed SNR means that there exists a constellation of aggregate tag waveforms of size $2^{R M}$, with each member of the constellation representing an ensemble of $M$ waveforms of the specified time-bandwidth product transmitted individually by each of $M$ possible tags corresponding to one of $2^{R}$ possible tag IDs for each tag, such that all $M$ transmitted tag IDs of $R$ bits each can be decoded simultaneously with arbitrarily low probability of error. Unfortunately, this cannot actually be guaranteed for any value of $R$ that satisfies Equation (1). In essence, what is really defined by Equation (1) is the maximum possible number of bits per second per Hertz per tag $(R / B T)$ that can be decoded given a particular average SNR per second per Hertz per tag ( $S N R / B T$ ), and tag constellations achieving any rate less than the maximum with arbitrarily low probability of error are guaranteed to exist only for sufficiently large time-bandwidth products. Hence, to find tag constellations with good collision resolution properties and a bit-rate per tag that satisfies Equation (1), one can expect to need large time-bandwidth products.

Secondly, Equation (1) can be rewritten as

$$
R<\frac{B T}{M \ln 2} \ln \left(1+\frac{M \cdot S N R}{B T}\right) \leq \frac{S N R}{\ln 2}
$$

with equality if and only if $S N R=0$. Furthermore, for sufficiently small values of the quantity $M \cdot S N R / B T$, we have

$$
\frac{B T}{M \ln 2} \ln \left(1+\frac{M \cdot S N R}{B T}\right) \approx \frac{S N R}{\ln 2}
$$

Hence, for all positive values of SNR, the achievable bitrate per tag is strictly less than $S N R / \ln 2$, and this level of efficiency (measured in terms of the achievable bit-rate per tag per unit of received SNR) can be achieved only if $B T>>M \cdot S N R$. Equivalently, the achievable bit-rate per tag will increase linearly with the SNR at the maximum possible rate of 1/ln 2 bits per tag per unit of SNR increase only as long as the time-bandwidth product of the tags is much greater than the quantity $M \cdot S N R$. Note that this is just another way of saying that the Shannon limit for RFID tags is $-1.6 \mathrm{~dB}$ of received SNR required in order to reliably transmit a single bit per tag over the channel (confer [12] or [14] for comparison), and that this level of efficiency can be maintained only as long as $B T>>M \cdot S N R$. This result is probably primarily of theoretical interest since detecting even a single known tag in the environment with an SNR of 100 (20 dB) at close to the Shannon limit of efficiency would require a time-bandwidth product close to 1000 .

Finally, the maximum achievable bit-rate given by Equation (1) is probably only achievable using detectors of unreasonable complexity and unreasonably large constellations of tag waveforms. However, a similar result [13] shows that a bit-rate of $R$ is achievable using more practical single-user detectors and an aggregate constellation of individual tag waveforms of size $M 2^{R}$ as long as

$$
R<(B T) \log _{2}\left(1+\frac{S N R}{B T+[M-1] S N R}\right)
$$

or

$$
M<\frac{2^{R / B T}}{2^{R / B T}-1}-\frac{B T}{S N R}
$$

In this case, the term " $R$ is an achievable bit-rate per tag" means that there exists an aggregate constellation of individual tag waveforms of size $M 2^{R}$, consisting of a different constellation of size $2^{R}$ for each of the possible tags, such that each tag can simultaneously transmit an arbitrary waveform from its constellation corresponding to one of $2^{R}$ possible tag IDs for each tag, and all $M$ transmitted tag IDs of $R$ bits each can be decoded with arbitrarily low probability of error. Once again, such constellations are guaranteed to exist only for sufficiently large time-bandwidth products.

Equations (3) and (4) are probably of much more practical use than Equations (1) and (2) in terms of determining how well a particular tag/interrogator combination is performing relative to a theoretical baseline. Once again, for sufficiently large values of $B T$ relative to $M \cdot S N R$, Equation (3) simplifies to

$$
R<\frac{S N R}{\ln 2},
$$

so using practical detectors results in no penalty with respect to maximum efficiently achievable bit-rate even though there 
may be a large penalty with respect to true maximum achievable bit-rate.

As an example of what all of this means, suppose we have a constellation of $2^{40}$ tag waveforms (i.e., 40-bit tag IDs) with bandwidth of $80 \mathrm{MHz}$ and duration $2 \mu$ s. If we design an interrogator to look for all $2^{40}$ possible tag IDs, then assuming that the temperature, strain, range, etc. of all the tags are known and each has a received SNR of $20 \mathrm{~dB}$, the true maximum number of tags that can be reliably detected in the environment simultaneously, as given by finding the largest integer satisfying Equation (2), is 12. Using Equation (4) instead to give an estimate of the maximum number of tags that can be detected using practical detectors leads to a bound of only 4 . Hence, one can really only expect to be able to detect approximately 4 tags at once from the entire constellation of such tags using an interrogator of reasonable complexity. On the other hand, if we can reduce the number of tags that the interrogator must search for to a mere $2^{20}$, then the true maximum number of detectable tags becomes 25 and the number of practically detectable tags becomes 10. Reducing the number of tags in the constellation even further to only $2^{10}$ gives a true maximum of 65 and a practical maximum of 21. Finally, if we want to be able to read all of the tags in the environment simultaneously (i.e., letting $M=2^{R}$ in Equations (2) and (4) ) then we get a true value of $M=145$ and a practical value of $M=41$.

Naturally, attempting to sense any telemetry with the tags in addition to identifying the tag IDs reduces all of these numbers considerably, so it is clear that there is a great benefit to keeping the number of tags that must be recognized by the interrogator to a minimum when attempting to use RFID tags for sensing applications.

\section{DISCUSSION OF THEORETICAL RESULTS}

As a practical matter, one never knows all of the parameters such as temperature, strain, and range that modulate the tag waveforms received at the reader. Indeed, in most scenarios, one is interested in estimating one or more of these parameters over some fixed range for each of the tags in the environment. Also, of course, in practical situations, the energy per tag received at the reader is never identical for all tags, nor is it known by the receiver, and the number of tags in the environment is also frequently not known by the receiver. Finally, the information-theoretic bounds derived in Section III are only guaranteed to be asymptotically achievable in situations where the data is encoded into discrete known codewords at the transmitter, where each distinct codeword corresponds to a known combination of tag ID and quantized values of the telemetry data of interest.

Hence, some interpretation of the theoretical results is needed in order to generate performance bounds corresponding to approximately optimal performance in more practical situations. From the point of view of determining a priori what performance one could hope to achieve from any SAW system subject to a given set of constraints on time-bandwidth product and SNR, the results given in Section III are sufficient. That is, they can be applied directly to answer questions such as: how many tags could possibly be detected simultaneously with arbitrarily low probability of error subject to the given constraints if the interrogator is programmed to search for $N$ known tags and estimate temperate using $M$ bits and range using $K$ bits? On the other hand, it is not very straightforward to determine how to apply the same results as benchmarks for performance evaluation of a known system. The problem is that the SAW tags themselves modulate the transmitted waveform in a continuous (analog) fashion determined by the physical environment, and this makes it very difficult to determine how many useful bits of telemetry are really being transmitted in each tag. In essence, infinitely many bits (or at least an extremely large number) are encoded in each waveform, and the corresponding information-theoretic results imply that no useful information can be transmitted reliably by attempting to decode all of that information at the interrogator.

Furthermore, within the class of waveforms that satisfies the constraints on time-bandwidth product and SNR, any particular set of waveforms will lead to different bounds on the minimum mean-squared-error (MSE) that can possibly be achieved when estimating parameters such as tag temperature and tag range from the tags. While these minimum MSE bounds can be used to estimate how many useful bits of information regarding the parameters of interest (i.e., parameter resolution) can possibly be transmitted from the tag to the receiver, the fact that they depend on the particular choice of waveforms means that it is difficult (or impossible) to identify any sort of fundamental limits on parameter resolution that depend only on timebandwidth product and SNR.

For our purposes, we would like to identify performance bounds that allow one to determine whether a particular combination of tag ID waveforms and interrogator algorithms (i.e., the system of interest) performs well in comparison to the best possible performance that one could expect from any possible set of waveforms interrogated with an optimal algorithm subject to the same constraints on timebandwidth product, SNR, number of tags in the environment, bounds on tag temperature, bounds on tag range, etc. satisfied by the system of interest. Toward that end, we suggest the following performance evaluation procedure:

1. Evaluate the system by fixing all relevant parameters such as temperature and range to determine performance relative to the theoretical bounds given by Equations (3) and (4) in Section III. This establishes performance relative to a true set of fundamental benchmarks.

2. Determine a set of tight lower bounds on the MSE for relevant parameters such as temperature and range using the known system tag waveforms. ${ }^{1}$ Evaluate system parameter estimation performance relative to these lower bounds. This establishes how well the

\footnotetext{
${ }^{1}$ This step can sometimes be quite computationally cumbersome.
} 
interrogator algorithms perform relative to the minimum MSE performance achievable with the given tag waveforms.

3. Using the actual MSE achieved for relevant parameter estimation by the system, estimate the number of useful bits of parameter information transmitted per tag. Using this estimate of tag bit rate, compare the system performance to the theoretical bounds given by Equations (3) and (4) in Section III for a set of practical operational scenarios of interest.

\section{SOME SIMULATED PERFORMANCE RESULTS}

In this section, we present some simulated performance results using Steps 1 and 3 of the evaluation procedure suggested above. We omit the results of step 2 in this case because we are still trying to identify an appropriate set of tight lower bounds on MSE. For these simulations, we use a set of simulated tag waveforms that were generated using an experimental procedure under investigation at JSC subject to various time-bandwidth constraints. The usable bandwidth for all tags was fixed at $50 \mathrm{MHz}$, the SNR was fixed at 20 $\mathrm{dB}$, and the time duration of the waveform was varied to achieve the desired time-bandwidth product.

The Interrogation algorithm used in this study operates assuming a discrete Fourier transform (DFT) of the received signal as the basic observational model and performs detection and estimation processing using a multi-resolution signal representation technique called the continuous wavelet transform (CWT) [15]. The interrogation process is iterative and is based on a process called multistage interference cancellation (MIC) [12], which recursively refines the initial estimates and attempts to remove already detected tag waveforms from the original received signal prior to the next iteration of detection/estimation processing. This is an approximately optimal approach to achieving the bounds given by Equations (3) and (4) which is discussed in more detail in [1].

For this study, two different tag constellations were evaluated in each of two different operational scenarios. Tag Set 1 (TS1) had a relatively small time-bandwidth product of only 95 with a total constellation size of 64 possible different tag waveforms (i.e., 6-bit IDs). For TS1, the entire constellation of 64 tags was always searched by the interrogator to determine which subset of tags were present in the environment. Tag Set 2 (TS2) had a much larger timebandwidth product of 165 with a total constellation size of $2^{20}$ (i.e., 20-bit IDs). For TS2, a total of 256 randomly selected tags were always searched by the interrogator to determine which subset of those 256 were present in the environment, which means that 8 bits of ID information were transmitted by each tag.

Scenario 1, which is the only one that produces performance results that can be compared directly to the theoretical bounds given by Equations (3) and (4), assumes that all of the tags in the environment are at exactly the same range and same temperature, both of which are assumed to be known precisely. Clearly, this is not a practical scenario, but it does allow the tag bit-rate to be determined precisely and eliminates the complications resulting from temperatureinduced waveform distortion as well as uncertain tag delay. This corresponds to Step 1 in the proposed evaluation process. Note that in this scenario, as well as Scenario 2, there is no assumption of phase coherence in the received waveforms. That is, it is assumed that any tag response received at the interrogator may be modulated by an arbitrary complex amplitude, which implies that not only is the magnitude of the received waveform not known a priori, but neither is the absolute phase of the received signal.

Scenario 2 was at the opposite end of the spectrum in terms of uncertainty regarding tag temperature and time delay. In this case, the tag temperatures were randomly distributed over a range of $200^{\circ} \mathrm{C}$, and the delays were randomly distributed over a period of approximately $0.06 \mu \mathrm{s}$, which corresponds to a range spread of approximately 10 meters. This represents a fairly large temperature range but a very reasonable range spread, and it clearly reveals the dependence of performance on uncertainty regarding tag temperature and delay. In all cases, even though the tags were simulated to be at different temperatures and ranges, the SNR for all tags was fixed at the same level. In a practical scenario, this would not generally be true, but since we are interested in the effects of different waveform characteristics rather than SNR on performance, we held the SNR constant to eliminate that particular source of variation.

The performance results for the 4 combinations of tag set and operational scenario are given in Tables 1-4. The results for each scenario are summarized briefly below.

\section{A. Scenario 1}

The performance results under Scenario 1 are summarized in Tables 1-2 below. These data were generated by running $N \geq 100$ random realizations of each of $K$ different interrogation experiments for each of the two tag sets, where $K=22$ for TS1 and $K=28$ for TS2. That is, $N$ realizations were generated for the two experiments (TS1 and TS2) in which there was a single tag responding in the environment, $N$ realizations for the two experiments in which two tags were responding, and so forth up to $N$ realizations for the two experiments in which there were $K$ tags responding. The number $M$ of responding tags in the simulations was limited to the maximum value of $K$, which was chosen as the upper bound on the value of $M$ produced by Equation (4) above for each of the tag sets under Scenario 1 .

The results presented in Tables 1-2 include only probability of detection $\left(\mathrm{P}_{\mathrm{d}}\right)$ and probability of false alarm $\left(\mathrm{P}_{\mathrm{fa}}\right)$, since the correct tag temperature and correct tag delay were provided as side information to the interrogation algorithm. The two detection probabilities were computed as follows:

- $\mathrm{P}_{\mathrm{d}}$ was computed by dividing the number of correctly detected tags in each experiment by the total number of tags responding in the experiment. If a tag was detected that was not actually a component of the simulated received signal, that detection was counted as a false alarm. 
- $\mathrm{P}_{\mathrm{fa}}$ was computed by dividing the number of false alarms in the experiment by the size of the number of tags in the scanning set exclusive of the responding tags. That is, if $M$ tags were responding, then it was possible for the interrogator to generate at most $L-M$ false alarms for that experiment, where $L=64$ for TS1 and $L=256$ for TS2.

Table 1. Scenario 1 Performance Results for TS1

\begin{tabular}{|c|c|c|c|c|c|}
\hline $\boldsymbol{M}$ & $\mathbf{P}_{\mathbf{d}}$ & $\mathbf{P}_{\mathbf{f a}}$ & $\boldsymbol{M}$ & $\mathbf{P}_{\mathbf{d}}$ & $\mathbf{P}_{\mathbf{f a}}$ \\
\hline $\mathbf{1}$ & $1.0000 \mathrm{E}+00$ & $0.0000 \mathrm{E}+00$ & $\mathbf{1 2}$ & $1.0000 \mathrm{E}+00$ & $4.6154 \mathrm{E}-03$ \\
\hline $\mathbf{2}$ & $1.0000 \mathrm{E}+00$ & $0.0000 \mathrm{E}+00$ & $\mathbf{1 3}$ & $1.0000 \mathrm{E}+00$ & $6.9608 \mathrm{E}-03$ \\
\hline $\mathbf{3}$ & $1.0000 \mathrm{E}+00$ & $0.0000 \mathrm{E}+00$ & $\mathbf{1 4}$ & $1.0000 \mathrm{E}+00$ & $9.2000 \mathrm{E}-03$ \\
\hline $\mathbf{4}$ & $1.0000 \mathrm{E}+00$ & $0.0000 \mathrm{E}+00$ & $\mathbf{1 5}$ & $1.0000 \mathrm{E}+00$ & $1.3878 \mathrm{E}-02$ \\
\hline $\mathbf{5}$ & $1.0000 \mathrm{E}+00$ & $0.0000 \mathrm{E}+00$ & $\mathbf{1 6}$ & $9.9531 \mathrm{E}-01$ & $1.7708 \mathrm{E}-02$ \\
\hline $\mathbf{6}$ & $1.0000 \mathrm{E}+00$ & $0.0000 \mathrm{E}+00$ & $\mathbf{1 7}$ & $9.9500 \mathrm{E}-01$ & $2.6809 \mathrm{E}-02$ \\
\hline $\mathbf{7}$ & $1.0000 \mathrm{E}+00$ & $0.0000 \mathrm{E}+00$ & $\mathbf{1 8}$ & $9.9056 \mathrm{E}-01$ & $2.9783 \mathrm{E}-02$ \\
\hline $\mathbf{8}$ & $1.0000 \mathrm{E}+00$ & $8.9286 \mathrm{E}-05$ & $\mathbf{1 9}$ & $9.8211 \mathrm{E}-01$ & $3.9667 \mathrm{E}-02$ \\
\hline $\mathbf{9}$ & $1.0000 \mathrm{E}+00$ & $6.3636 \mathrm{E}-04$ & $\mathbf{2 0}$ & $9.6750 \mathrm{E}-01$ & $4.6023 \mathrm{E}-02$ \\
\hline $\mathbf{1 0}$ & $1.0000 \mathrm{E}+00$ & $1.6667 \mathrm{E}-03$ & $\mathbf{2 1}$ & $9.5905 \mathrm{E}-01$ & $4.9767 \mathrm{E}-02$ \\
\hline $\mathbf{1 1}$ & $1.0000 \mathrm{E}+00$ & $2.5472 \mathrm{E}-03$ & $\mathbf{2 2}$ & $9.2932 \mathrm{E}-01$ & $5.5833 \mathrm{E}-02$ \\
\hline
\end{tabular}

Table 2. Scenario 1 Performance Results for TS2

\begin{tabular}{|c|c|c|c|c|c|}
\hline $\boldsymbol{M}$ & $\mathbf{P}_{\mathbf{d}}$ & $\mathbf{P}_{\mathbf{f a}}$ & $\boldsymbol{M}$ & $\mathbf{P}_{\mathbf{d}}$ & $\mathbf{P}_{\mathbf{f a}}$ \\
\hline $\mathbf{1}$ & $1.0000 \mathrm{E}+00$ & $0.0000 \mathrm{E}+00$ & $\mathbf{1 5}$ & $1.0000 \mathrm{E}+00$ & $5.9277 \mathrm{E}-04$ \\
\hline $\mathbf{2}$ & $1.0000 \mathrm{E}+00$ & $0.0000 \mathrm{E}+00$ & $\mathbf{1 6}$ & $1.0000 \mathrm{E}+00$ & $8.3333 \mathrm{E}-04$ \\
\hline $\mathbf{3}$ & $1.0000 \mathrm{E}+00$ & $0.0000 \mathrm{E}+00$ & $\mathbf{1 7}$ & $1.0000 \mathrm{E}+00$ & $1.3150 \mathrm{E}-03$ \\
\hline $\mathbf{4}$ & $1.0000 \mathrm{E}+00$ & $0.0000 \mathrm{E}+00$ & $\mathbf{1 8}$ & $1.0000 \mathrm{E}+00$ & $2.2809 \mathrm{E}-03$ \\
\hline $\mathbf{5}$ & $1.0000 \mathrm{E}+00$ & $0.0000 \mathrm{E}+00$ & $\mathbf{1 9}$ & $1.0000 \mathrm{E}+00$ & $2.6522 \mathrm{E}-03$ \\
\hline $\mathbf{6}$ & $1.0000 \mathrm{E}+00$ & $0.0000 \mathrm{E}+00$ & $\mathbf{2 0}$ & $9.9667 \mathrm{E}-01$ & $4.6005 \mathrm{E}-03$ \\
\hline $\mathbf{7}$ & $1.0000 \mathrm{E}+00$ & $0.0000 \mathrm{E}+00$ & $\mathbf{2 1}$ & $1.0000 \mathrm{E}+00$ & $5.5927 \mathrm{E}-03$ \\
\hline $\mathbf{8}$ & $1.0000 \mathrm{E}+00$ & $0.0000 \mathrm{E}+00$ & $\mathbf{2 2}$ & $1.0000 \mathrm{E}+00$ & $5.6980 \mathrm{E}-03$ \\
\hline $\mathbf{9}$ & $1.0000 \mathrm{E}+00$ & $3.8558 \mathrm{E}-05$ & $\mathbf{2 3}$ & $1.0000 \mathrm{E}+00$ & $6.9896 \mathrm{E}-03$ \\
\hline $\mathbf{1 0}$ & $1.0000 \mathrm{E}+00$ & $3.8715 \mathrm{E}-05$ & $\mathbf{2 4}$ & $1.0000 \mathrm{E}+00$ & $7.5944 \mathrm{E}-03$ \\
\hline $\mathbf{1 1}$ & $1.0000 \mathrm{E}+00$ & $1.9436 \mathrm{E}-04$ & $\mathbf{2 5}$ & $1.0000 \mathrm{E}+00$ & $1.0472 \mathrm{E}-02$ \\
\hline $\mathbf{1 2}$ & $1.0000 \mathrm{E}+00$ & $3.9032 \mathrm{E}-05$ & $\mathbf{2 6}$ & $9.9597 \mathrm{E}-01$ & $1.2629 \mathrm{E}-02$ \\
\hline $\mathbf{1 3}$ & $1.0000 \mathrm{E}+00$ & $3.1354 \mathrm{E}-04$ & $\mathbf{2 7}$ & $9.9083 \mathrm{E}-01$ & $1.3100 \mathrm{E}-02$ \\
\hline $\mathbf{1 4}$ & $1.0000 \mathrm{E}+00$ & $3.5419 \mathrm{E}-04$ & $\mathbf{2 8}$ & $9.8639 \mathrm{E}-01$ & $1.7084 \mathrm{E}-02$ \\
\hline
\end{tabular}

As the results in Tables 1-2 indicate, for this scenario, in which there is no uncertainty regarding the tag delay or temperature, the waveforms corresponding to TS2 give nearly optimal performance but the waveforms corresponding to TS1 do not perform as well. Since the only significant difference between the two sets of simulated tag waveforms was the considerably larger time-bandwidth product of TS2, this clearly demonstrates the advantage of choosing tags with a large time-bandwidth product. That is, not only is it possible to reliably detect more tags simultaneously using TS2 than TS1, but the number of reliably detected tags using TS2 comes much closer to the upper bound given by Equation (4) than when using TS1.

\section{B. Scenario 2}

The performance results under Scenario 2 are summarized in Tables 3-4 below. The results presented in Tables 3-4 again include both $\mathrm{P}_{\mathrm{d}}$ and $\mathrm{P}_{\mathrm{fa}}$, but for this scenario, estimates of the MSE for temperature $\left(\sigma_{\text {temp }}\right.$ in $\left.{ }^{\circ} \mathrm{C}\right)$ and range ( $\sigma_{\text {range }}$ in meters) for detected tags were also computed and reported. Again, these data were generated by running $N \geq 100$ random realizations of each of $K$ different interrogation experiments for each of the two tag sets, where $K=12$ for TS1 and $K=15$ for TS2. In this case, the value of $K$ was chosen as a rough estimate of the upper bound on the value of $M$ produced by Equation (4). The estimate was computed assuming the number of bits of temperature information transmitted by each tag was $\log _{2}\left(200 / 2 \sigma_{\text {temp }}\right)$, and the number of bits of useful range information was $\log _{2}\left(10 / 2 \sigma_{\text {range }}\right)$. The exact bound cannot be determined for this scenario because both the temperature and delay of the tag response must be estimated along with the tag ID, which makes it impossible to determine a true data rate for the tags. The estimated upper bounds for TS1 and TS2 under Scenario 2 are 8 and 12, respectively.

Table 3. Scenario 2 Performance Results for TS1

\begin{tabular}{|c|c|c|c|c|}
\hline $\boldsymbol{M}$ & $\mathbf{P}_{\mathbf{d}}$ & $\mathbf{P}_{\mathbf{f a}}$ & $\sigma_{\text {temp }}$ & $\sigma_{\text {range }}$ \\
\hline $\mathbf{1}$ & $1.0000 \mathrm{e}+00$ & $0.0000 \mathrm{E}+00$ & $1.2737 \mathrm{E}+00$ & $6.3446 \mathrm{E}-01$ \\
\hline $\mathbf{2}$ & $1.0000 \mathrm{e}+00$ & $0.0000 \mathrm{E}+00$ & $1.1815 \mathrm{E}+00$ & $6.3180 \mathrm{E}-01$ \\
\hline $\mathbf{3}$ & $1.0000 \mathrm{e}+00$ & $0.0000 \mathrm{E}+00$ & $1.2415 \mathrm{E}+00$ & $6.5276 \mathrm{E}-01$ \\
\hline $\mathbf{4}$ & $1.0000 \mathrm{e}+00$ & $0.0000 \mathrm{E}+00$ & $1.1847 \mathrm{E}+00$ & $6.4708 \mathrm{E}-01$ \\
\hline $\mathbf{5}$ & $1.0000 \mathrm{e}+00$ & $0.0000 \mathrm{E}+00$ & $1.2242 \mathrm{E}+00$ & $6.4012 \mathrm{E}-01$ \\
\hline $\mathbf{6}$ & $1.0000 \mathrm{e}+00$ & $9.3703 \mathrm{E}-05$ & $1.2987 \mathrm{E}+00$ & $6.4919 \mathrm{E}-01$ \\
\hline $\mathbf{7}$ & $1.0000 \mathrm{e}+00$ & $1.9069 \mathrm{E}-04$ & $1.3937 \mathrm{E}+00$ & $6.3379 \mathrm{E}-01$ \\
\hline $\mathbf{8}$ & $1.0000 \mathrm{e}+00$ & $5.8230 \mathrm{E}-04$ & $1.3931 \mathrm{E}+00$ & $6.5072 \mathrm{E}-01$ \\
\hline $\mathbf{9}$ & $1.0000 \mathrm{e}+00$ & $2.9644 \mathrm{E}-04$ & $1.4522 \mathrm{E}+00$ & $6.4558 \mathrm{E}-01$ \\
\hline $\mathbf{1 0}$ & $1.0000 \mathrm{e}+00$ & $2.0129 \mathrm{E}-03$ & $1.4886 \mathrm{E}+00$ & $6.3861 \mathrm{E}-01$ \\
\hline $\mathbf{1 1}$ & $1.0000 \mathrm{e}+00$ & $3.6916 \mathrm{E}-03$ & $1.5308 \mathrm{E}+00$ & $6.4659 \mathrm{E}-01$ \\
\hline $\mathbf{1 2}$ & $9.9366 \mathrm{e}-01$ & $6.4799 \mathrm{E}-03$ & $2.2925 \mathrm{E}+00$ & $6.4883 \mathrm{E}-01$ \\
\hline
\end{tabular}


Table 4. Scenario 2 Performance Results for TS2

\begin{tabular}{|c|c|c|c|c|}
\hline $\boldsymbol{M}$ & $\mathbf{P}_{\mathbf{d}}$ & $\mathbf{P}_{\mathbf{f a}}$ & $\sigma_{\text {temp }}$ & $\sigma_{\text {range }}$ \\
\hline $\mathbf{1}$ & $1.0000 \mathrm{E}+00$ & $0.0000 \mathrm{E}+00$ & $7.5242 \mathrm{E}-01$ & $6.0932 \mathrm{E}-01$ \\
\hline $\mathbf{2}$ & $1.0000 \mathrm{E}+00$ & $0.0000 \mathrm{E}+00$ & $6.8908 \mathrm{E}-01$ & $6.4922 \mathrm{E}-01$ \\
\hline $\mathbf{3}$ & $1.0000 \mathrm{E}+00$ & $0.0000 \mathrm{E}+00$ & $6.7555 \mathrm{E}-01$ & $6.3885 \mathrm{E}-01$ \\
\hline $\mathbf{4}$ & $1.0000 \mathrm{E}+00$ & $0.0000 \mathrm{E}+00$ & $6.6402 \mathrm{E}-01$ & $6.4074 \mathrm{E}-01$ \\
\hline $\mathbf{5}$ & $1.0000 \mathrm{E}+00$ & $0.0000 \mathrm{E}+00$ & $6.9764 \mathrm{E}-01$ & $6.3432 \mathrm{E}-01$ \\
\hline $\mathbf{6}$ & $1.0000 \mathrm{E}+00$ & $3.8835 \mathrm{E}-05$ & $7.0200 \mathrm{E}-01$ & $6.2395 \mathrm{E}-01$ \\
\hline $\mathbf{7}$ & $1.0000 \mathrm{E}+00$ & $3.8991 \mathrm{E}-05$ & $7.1581 \mathrm{E}-01$ & $6.2721 \mathrm{E}-01$ \\
\hline $\mathbf{8}$ & $1.0000 \mathrm{E}+00$ & $0.0000 \mathrm{E}+00$ & $6.7153 \mathrm{E}-01$ & $6.4966 \mathrm{E}-01$ \\
\hline $\mathbf{9}$ & $1.0000 \mathrm{E}+00$ & $0.0000 \mathrm{E}+00$ & $7.0092 \mathrm{E}-01$ & $6.4469 \mathrm{E}-01$ \\
\hline $\mathbf{1 0}$ & $1.0000 \mathrm{E}+00$ & $0.0000 \mathrm{E}+00$ & $7.2374 \mathrm{E}-01$ & $6.5102 \mathrm{E}-01$ \\
\hline $\mathbf{1 1}$ & $1.0000 \mathrm{E}+00$ & $0.0000 \mathrm{E}+00$ & $7.7681 \mathrm{E}-01$ & $6.4138 \mathrm{E}-01$ \\
\hline $\mathbf{1 2}$ & $1.0000 \mathrm{E}+00$ & $0.0000 \mathrm{E}+00$ & $7.3274 \mathrm{E}-01$ & $6.3473 \mathrm{E}-01$ \\
\hline $\mathbf{1 3}$ & $1.0000 \mathrm{E}+00$ & $3.9954 \mathrm{E}-05$ & $7.5866 \mathrm{E}-01$ & $6.5109 \mathrm{E}-01$ \\
\hline $\mathbf{1 4}$ & $1.0000 \mathrm{E}+00$ & $2.0059 \mathrm{E}-04$ & $7.4054 \mathrm{E}-01$ & $6.4851 \mathrm{E}-01$ \\
\hline $\mathbf{1 5}$ & $1.0000 \mathrm{E}+00$ & $2.8200 \mathrm{E}-04$ & $7.5413 \mathrm{E}-01$ & $6.5084 \mathrm{E}-01$ \\
\hline & & & & \\
\hline
\end{tabular}

As Tables 3-4 indicate, both tag sets give very reliable detection of tags even above the rough estimates of the upper bounds that we computed. Also, TS2 once again outperforms TS1, in this case in terms of both number of reliably detectable tags and temperature estimation performance; however, the difference in performance is not as obvious in this case as it is under Scenario 1. Finally, the range estimation performance of both tag sets is very similar. This is not really surprising since, for example, the Cramer-Rao lower bound on range estimate variance is known to depend only on bandwidth and SNR, not on time-bandwidth product [16].

To get a much clearer picture of what is going on vis-àvis the upper bounds given by Equations (3) and (4) in this case, one would ideally need to devise a different set of performance simulations or at the very least run a much larger number of trials in the current simulation. Nevertheless, closer inspection of the results in Tables 3-4, particularly the point at which $\mathrm{P}_{\mathrm{fa}}$ begins to increase steadily, indicates that performance begins to deteriorate for TS1 when there are more than 7 tags responding in the environment but not until there are approximately 13 tags responding for TS2. This is fairly consistent with the upper bound estimates of 8 and 12 and once again indicates that the increased time-bandwidth product of TS2 provides a significant benefit, not only in terms of improving the collision resolution and temperature estimation performance, but also in terms of approaching the bounds on achievable data rates per tag.

\section{CONCLUSION}

In this paper, we have derived some fundamental bounds on the information capacity and collision resolution for SAW
RFID tags. Using simulated waveforms based on tag waveform design procedures under investigation at JSC, we have also demonstrated that practical tags can perform well with respect to these bounds and that tag performance in terms of both collision resolution and temperature estimation improves significantly as the time-bandwidth product of the tags increases.

\section{REFERENCES}

[1] R. J. Barton, T. F. Kennedy, R. M. Williams, P. W. Fink, P. H. Ngo, and R. R. Ingle, "Detection, Identification, Location, and Remote Sensing using SAW RFID Sensor Tags," in 2010 IEEE Aerospace Conference Big Sky, Montana, 2010, pp. 1-19.

[2] J. Dowling, M. M. Tentzeris, and N. Becket, "RFID-Enabled Temperature Sensing Devices: A Major Step Forward for Energy Efficiency in Home and Industrial Applications?," in IEEE MTT-S International Microwave Workshop on Wireless Sensing, Local Positioning, and RFID, 2009, pp. 1-4.

[3] G. Fischerauer, "Surface Acoustic Wave Devices," in Sensors. A Comprehensive Survey. vol. 8, W. Gopel, J. Hesse, J. N. Zemel, H. Meixner, and R. Jones, Eds.: Weinheim: VCH, 1995.

[4] J.-G. Oh, B. Choi, and S.-Y. Lee, "SAW based passive sensor with passive signal conditioning using MEMS A/D converter," Sensors and Actuators A: Physical, vol. 141, pp. 631-639, February 2008.

[5] J. K. Perng, W. D. Hunt, and P. J. Edmonson, "Development of a Shear Horizontal SAW RFID Biosensor," in 2007 IEEE Sensors Conference, 2007, pp. 691-694.

[6] A. Saitoh and T. Nomura, "Wireless Sensor System Using Surface Acoustic Wave Devices," in 2009 ICCAS-SICE, 2009, pp. 23592363.

[7] A. Steltzer, S. Scheiblhofer, S. Schuster, and M. Brandl, "Multi Reader/Multi-Tag SAW RFID Systems Combining Tagging, Sensing, and Ranging for Industrial Applications," in 2008 IEEE International Frequency Control Symposium, 2008, pp. 263-272.

[8] S. Harma, W. G. Arthur, C. S. Hartmann, R. G. Maev, and V. P. Plessky, "Inline SAW RFID Tag Using Time Position and Phase Encoding," IEEE Transactions on Ultrasonics, Ferroelectrics, and Frequency Control, vol. 55, pp. 1840-1846, August 2008.

[9] V. P. Plessky and L. M. Reindl, "Review on SAW RFID tags," IEEE Transactions on Ultrasonics, Ferroelectrics, and Frequency Control, vol. 57, pp. 654-668, 2010.

[10] W. C. Wilson, D. C. Malocha, N. Kozlovski, D. R. Gallagher, B. Fisher, J. Pavlina, N. Saldanha, D. Puccio, and G. M. Atkinson, "Orthogonal Frequency Coded SAW Sensors for Aerospace SHM Applications," IEEE Sensors Journal, vol. 9, pp. 1546-1556, November 2009.

[11] C. S. Hartmann and L. T. Claiborne, "Fundamental Limitations on Reading Range of Passive IC-Based RFID and SAW-Based RFID," in 2007 IEEE International Conference on RFID Grapevine, TX, 2007, pp. 41-48

[12] J. G. Proakis, Digital Communications, Fourth ed. Boston, MA: McGraw-Hill, 2001.

[13] T. M. Cover and J. A. Thomas, Elements of Information Theory, Second ed. Hoboken, NJ: John Wiley \& Sons, 2006.

[14] S. Verdu, "On Channel Capacity Per Unit Cost," IEEE Transactions on Information Theory, vol. 36, pp. 1019-1030, September 1990.

[15] I. Daubechies, Ten Lectures on Wavelets. Philadelphia: SIAM, 1992.

[16] Q. Jin, K. M. Wong, and Z.-Q. Luo, "The Estimation of Time Delay and Doppler Stretch of Wideband Signals," IEEE Transactions on Signal Processing, vol. 43, pp. 904-916, 1995. 